\title{
Conceptos básicos del paradigma de la complejidad aplicados a la cuestión del método en Psicología Social
}

\author{
The paradigm of complexity: Conceptual basis applied to Social \\ Psychology method
}

\author{
María de la Villa Moral Jiménez* \\ Universidad de Oviedo, España \\ (Rec.: septiembre de 2015 - Acept.: marzo de 2016)
}

\begin{abstract}
Resumen
El objetivo del presente artículo es reflexionar sobre las aportaciones del paradigma de la complejidad (PC) dentro del debate epistemológico actual y su aplicación a la cuestión del método en psicología social, ya que representa una descripción más ajustada de los procesos psicosociales. Se parte de la consideración de que la realidad es constitutivamente compleja en sus manifestaciones. Dadas las propiedades irreductibles en sus diversas facetas no sólo físicas, sino también psíquicas y sociales así como, las interrelaciones e interconexiones de los constituyentes psicológicos, sociales, biológicos, económicos, políticos, culturales y ecológicos, ha se sugerirse una nueva manera de abordar su análisis integral acorde a los planteamientos de las orientaciones emergentes en psicología social. Se resalta la necesidad de evitar la habitual simplificación en la que se incurre con la aportación de explicaciones lineales del comportamiento social desde modelos parsimoniosos. De este modo, se demanda una Psicología Social que, desde la asunción de la complejidad del ser humano y el estudio integral de tales implicaciones, colabore en el proyecto de comprenderlo. Asimismo, se defiende una metodología compleja, dinámica y no lineal adaptada a la variabilidad del objeto de estudio de la psicología social desde una base transdisciplinaria, auténticamente plural, crítica e integradora.
\end{abstract}

Palabras clave: psicología social, paradigma de la complejidad, método, transdisciplinariedad.

\begin{abstract}
This article aims to reflect on the contributions of the Paradigm of Complexity (PC) to the current epistemological debate, and its significance in the question of method in social psychology for it represents a more accurate description of the psychosocial processes. It begins with the consideration that reality is constitutively complex in its manifestations. A new way of addressing a thorough analysis according to the approaches of emerging orientations in social psychology arises, given the not only physical, but also psychic and social irreducible properties, along with the interrelations and interconnections between the psychological, social, biological, economic, political, cultural and ecological constituents. Similarly, it is important to avoid common simplifications by providing linear explanations of social behavior from parsimonious models. Thus, this investigation demands a new social psychology that assumes the complexity of human beings as well as the comprehensive study of those implications to improve its understanding. Similarly, the study arises the need of a complex, dynamic and nonlinear methodology adapted to the variability of the social psychology subject matter from a trans-disciplinary, genuinely plural, critical and inclusive model.
\end{abstract}

Keywords: social psychology, paradigm of complexity, method, transdisciplinarity.

\footnotetext{
“Correspondencia a: María de la Villa Moral Jiménez. Dirección postal: Universidad de Oviedo, Calle San Francisco 1 (33003), Oviedo, España. E-mail: mvilla@uniovi.es.
} 
"Construimos nuestras obras de conocimiento como casas de techo, como si el conocimiento no lo fuera a cielo raso; continuamos haciendo obras clausuradas, cerradas al futuro que hará surgir lo nuevo y lo desconocido, y nuestras conclusiones aportan la respuesta segura a la interrogación inicial, con, únicamente in extremis, en las obras universitarias, algunas interrogaciones nuevas".

Edgard Morin.

\section{Introducción}

Desde la controvertida crisis de relevancia de la psicología social, diversas orientaciones incipientes caracterizan el proceso de reemergencia de esta disciplina. Hace más de dos décadas en el análisis de lbáñez (1994) se argumentaba que, tras una fase de asentamiento, los psicólogos sociales se han interesado en indagar las características de la propia tradición cultural, actuando por contraste con la especificidad norteamericana como adalid de una disciplina individualista, experimentalista y psicologuita defendida desde la historiografía oficial de la disciplina (Kelly, 1981; Klappenbach, 2006; Lefebvre, 1974; Woodward, 1980). En concreto, ni la psicología social latinoamericana ni la europea contaban con un pasado suficientemente abonado para que cristalizasen los presupuestos individualistas y positivistas característicos de la perspectiva psicosocial dominante. Teniendo en cuenta sus orientaciones más colectivistas del pasado, el estallido e impacto de la crisis que sacudió a la psicología social norteamericana fue menor. En este sentido, Graumann (1990) anticipaba que el proceso de creciente europeización de la psicología social en las últimas décadas "ha servido para generar una interacción más viva y frecuente entre los psicólogos" (p. 34). Precisamente, convenimos con Montero (2010) en que si algo distingue a un tipo de psicología social latinoamericana es su inclinación hacia la crítica y su práctica, asumiéndose la complejidad como una característica de la crítica que, también es consustancialmente analéctica, dinámica y persistente en su oposición al principio de autoridad.

La atemporalidad y ahistoricismo, la distancia paradigmática, el predominio del método hipotéticodeductivo, el empleo generalizado del modelo metodológico experimentalista, así como la suposición de la existencia de variables homeostáticas constituyen las características básicas del paradigma que ha dominado la psicología social desde sus orígenes. Sin embargo, el talante más crítico del pensamiento social europeo y latinoamericano ha influido en la emergencia de orientaciones críticas diversificadas (Ovejero \& Ramos, 2011). A un nivel sistemático, a través de la revisión de las publicaciones de las cinco ediciones del Handbook of Social Psychology (Fiske, Gilbert \& Lindzey, 2010) se pueden identificar el listado de temas que han sido y son dominantes en la psicología social. Cabe citar refundaciones singularizadas de diversas orientaciones hasta derivar en las formulaciones del neodarwinismo, freudomarxismo, sociocognitivismo, microsociología y etnometodología, etc.

La realidad es sumamente compleja (Gell-Mann, 1998; Gribbin, 2006; Lewin, 1997; Prigogine, 1997), lo cual es defendido desde el Paradigma de la Complejidad (PC) que se propone como paradigma alternativo a interpretaciones reduccionistas en diversas disciplinas, entre ellas las ciencias sociales, orientadas hacia un conocimiento comprehensivo del ser humano. La realidad es interpretable desde muy diversos posicionamientos, de modo que la complejidad de analizar la realidad también puede justificarse desde la evidencia de que las descripciones en ciencias sociales no son en manera alguna descripciones simples ni neutras, sino que resultan de nuestro diálogo con la realidad, de nuestra construcción social de la misma y de nuestros modos de acción e investigación. Específicamente, los fenómenos objeto de estudio de la psicología social son propios de procesos de sincretismo sociocultural aplicables a un nuevo campo de la experiencia y del conocimiento, en cuyas complejidades el objeto es comprendido de formas múltiples y diversas, a través de una actitud cognoscente hacia el mundo (Morin, 1994, 1995, 1999, 2001). Si hay un reconocimiento de la complejidad de las realidades objeto de análisis resulta sorprendente que todavía prevalezca la visión de reducir lo complejo hasta desvirtuar su esencia y existencia, lo cual conduce a fragmentar la realidad y a parcializar los saberes. Así, las teorías del PC representan una nueva perspectiva epistemológica -no reduccionista, sino transdiciplinar, de aplicación en el estudio del comportamiento social (Luhmann, 1998; Munnè, 1995; Wagensberg, 1985). Ciertamente, existe una paradoja entre la certidumbre de las leyes de la naturaleza que se busca definir, y la incertidumbre y la inestabilidad de los hechos reales. Orden y desorden, equilibro y desequilibrio, certidumbre e incertidumbre, determinismo y azar, linealidad y nolinealidad, estático y dinámico, organización y caos son apenas algunos de los conceptos relacionados con la complejidad. De este modo, se demanda una psicología social que, desde la asunción de la complejidad del ser humano y el estudio integral de tales implicaciones, colabore en el proyecto de estudiarlo en profundidad.

Son relativamente abundantes los trabajos que en la mayoría de los campos de la ciencia apuntan hacia un paradigma epistemológico basado en la complejidad como alternativa al paradigma dominante que rinde culto a la simplicidad. Esta concepción simplista del método científico desvirtúa en esencia el conocimiento de la realidad reduciendo su complejidad, objetivando sus subjetividades, incurriendo en reduccionismos metodológicos en el quehacer científico, interesándose por explicaciones causales deterministas, etc., sirviéndose de todo ello como instrumentos de dominio y poder.

El paradigma de la simplificación implica una visión de la realidad que restringe lo complejo a lo simple, reduce el conjunto a lo elemental, rechaza el desorden y potencia la disyunción (entre objetos y 
contexto, sujeto y objeto, saberes y disciplinas), como principales directrices. Si bien recordemos que Morin $(1994,1995)$ afirma que la complejidad no es su opuesto, sino la unión de la simplicidad y la complejidad, de ahí la conveniencia de conjugar ambas perspectivas (Luengo-González, 2008). Las descripciones en ciencias sociales no son simples ni neutras, sino que resultan de nuestro diálogo con la realidad, de nuestra construcción social de la misma y de nuestros modos de acción e investigación.

Como señala Munnè (2004), cabe preguntarse por qué domina un paradigma basado en la simplicidad cuando por el contrario la realidad es compleja. Para el conocimiento científico tradicionalmente dominante, admitir una realidad compleja podría representar una ambigüedad que trastoque sus principios de una realidad ordenada, simplificada y armónica. Debido a sus reticencias, se han seguido proponiendo unos estudios reduccionistas y fragmentarios, en cambio, el campo social ha de ser estudiado como un sistema complejo adaptativo.

Entre las aportaciones emergentes en psicología social destacan una serie de orientaciones teóricas que asumen la complejidad. Representan una ruptura con la visión neopositivista de la ciencia y cuestionan los fundamentos epistemológicos de lo que tradicionalmente se ha conceptualizado como conocimiento científico. Críticamente cuestionan el papel de lo racional en la producción de conocimiento o sobre la naturaleza misma de aquello que entendemos por Ciencia y Razón (críticos con la idea de progreso propia de la visión iluminista). Destaca una gran variedad de orientaciones, con comunalidades y divergencias sustanciales, que representan alternativas a los modelos teóricos institucionalizados en psicología social. Emergen diversas orientaciones, tendencias y metateorías, como el posestructuralismo, el construccionismo social, la psicología social crítica, las ciencias sociales discursivas, la psicología social de la liberación y las perspectivas de análisis crítico feminista, como las más representativas.

Las explicaciones en ciencias sociales están estrechamente ligadas a determinadas concepciones sobre el ser humano que permanecen íntimamente conectadas con los arquetipos imperantes en las sociedades donde se (re) producen. De acuerdo con Munnè (2004), el conocimiento de la realidad viene condicionado por la propia epistemología. Ambos aspectos son relevantes en la medida en que contribuyen a la prescripción y estudio de lo que determina el comportamiento humano, a nivel individual, grupal y comunitario e, influye sobre la adecuación y licitud de las metodologías de investigación así como sobre la conveniencia de intervenir sobre ello.

Sin embargo, paradójicamente, para garantizar retóricamente el afán de cientificidad de su saber, se ha incurrido en reduccionismos teóricos y metodológicos. Coincidimos con Crespo (1995) en que buena parte de la psicología social ha estado fundamentada sobre una explicación del ser humano y su acción concebida desde el determinismo, el mecanicismo y el reduccionismo. Desde la psicología social no se pueden obviar tales condicionantes, en la medida en que se inscriben en su propia manera de producir conocimiento. Por ello, se incide en la conveniencia de adoptar renovados modelos interpretativos de la complejidad del ser humano y de sus realidades. Por tanto, nuestro objetivo no es otro que aplicar diversas nociones básicas del PC a cuestiones metodológicas planteadas en las orientaciones emergentes de la psicología social contemporánea.

\section{Planteamiento}

\section{Perspectivas de análisis nodulares del PC}

EI PC aglutina a expertos de diversos campos de conocimiento que insisten en la conveniencia de adoptar nuevos modelos epistemológicos en virtud de los cuales se construyan modelos interpretativos y metodologías más ajustados de la realidad. Dada la diversidad de disciplinas y posicionamientos encuadrados bajo este paradigma, se ha de evitar dar una imagen de unidad, dada la diversidad epistemológica subyacente. Frente al paradigma reduccionista dominante en investigación en los últimos años, las teorías de la complejidad (no linealidad, fractalidad, caoticidad, autoorganización, etc.) representan una perspectiva epistemológica alternativa y emergente como intento válido de superación del parsimonioso paradigma positivistacartesiano. Ciertamente, ya Munnè (1993, 1994, 1995, 2004, 2005, 2007) incidió magistralmente en la evidencia de que la realidad es mucho más compleja de lo que veníamos creyendo.

Desde esta perspectiva, el mundo es una compleja red de elementos relacionados, como una suerte de entramado, articulándose en torno a cuatro perspectivas teóricas: caoticidad, borrosidad, catastrofismo y fractalidad. La realidad a la que pertenecemos, que somos, se define por una compleja red de nudos entrelazados e interconectados entre sí. Fenómenos que influyen en otros aparentemente lejanos; drásticas situaciones en cursos de tiempo más estables; patrones caóticos; la provocación de la estabilidad mediante el cambio; la repetición de ciertos patrones de comportamiento; cambios cualitativos asociados a procesos de repetición similar, etc., representan fenómenos de sumo interés para el PC.

A modo de síntesis, algunos de los supuestos básicos del PC (véase una ampliación en Munnè, 2005; Pastor \& García-Izquierdo, 2007) son:

a) Como base matemática de la complejidad se acepta el principio de no linealidad (Poincaré, 1908/1948) que se refiere a la no proporcionalidad causa-efecto. De este modo, pequeñas perturbaciones pueden dar lugar a grandes cambios y viceversa, dada la interacción entre elementos del sistema, la cual puede desencadenar comportamientos muy complejos.

b) La caoticidad (Lorenz, 1965/1995) se refiere a la hipersensibilidad a las condiciones iniciales, de manera que cambios mínimos en las condiciones iniciales, propios de patrones caóticos 

$\begin{array}{ccc}\text { característicos de una dinámica no lineal } & \text { no } \\ \text { indeterminada, pueden resultar relevantes }\end{array}$ (Zimmerman, 1993).

c) La idea de equilibrios interrumpidos (Eldredge \& Gould, 1972) plantea una evolución no lineal: largos períodos de tiempo sin cambios perceptibles salpicados por breves, pero intensos, momentos súbitos de cambio que facilitan la aparición de nuevas propiedades.

d) La ambigüedad de los sistemas adaptativos complejos se asocia a las interacciones 0 fluctuaciones de los componentes del sistema.

e) La redefinición de la predicción y el control en términos de atractores, esto es, estados a los que tiende un sistema por su propia dinámica y en el que se asienta de una manera relativamente estable.

f) El catastrofismo (Thom, 1997) nos habla de cambios bruscos y repentinos que producen, irreversiblemente, un nuevo estado del sistema.

g) Por emergencia (Johnson, 2003) se entiende el surgimiento espontáneo de nuevas propiedades en el sistema como consecuencia de las interacciones entre sus componentes, de ahí que se aluda a un nivel de organización cualitativamente distinto y más complejo.

h) La autoorganización emergente se refiere a la génesis de orden, es decir, a la innovación y creación de nuevas formas y estructuras. Entendida como proceso emergente que acaba consolidando cierto orden, puede proponerse ya sea desde la biología, la autopoiesis (Maturana \& Varela, 1990, 1994), desde la termodinámica, las estructuras disipativas en sistemas alejados del equilibrio (Nicolis \& Prigogine, 1994; Prigogine, 1983, 1997) o desde el pensamiento filosófico, la recursividad (Morin, 1994).

i) Se entiende por fractales (Mandelbrot, 1987) las huellas geométricas que dejan los sistemas caóticos, a modo de patrones regulares de organización que indican un orden complejo en comportamientos aparentemente aleatorios. Representan la articulación entre lo que cambia y lo que permanece, al representar gráficamente los patrones que organizan un sistema complejo.

j) La teoría de los conjuntos borrosos y la lógica borrosa (Zadeh, 1965) propone que la pertenencia a un conjunto dado no es una cuestión dicotómica, sino de grado, sustituyéndose la probabilidad por la posibilidad. Así, desde el principio de incompatibilidad, se propone que a mayor complejidad, menor precisión y mayor borrosidad.

De forma pionera, la aplicación de metodología no lineal en psicología es deudora del trabajo de Lord y Novick (1968). Asimismo, los inicios de la aplicación de la teoría del caos a la explicación de los fenómenos sociales se encuentran en el trabajo precursor de Cronbach (1988). Poco a poco, la perspectiva de la complejidad se fue incorporando a la investigación psicológica $y$, posteriormente, se tiende a una refocalización a las ciencias de la vida (Byrne, 1998; Holland, 1995, 1998), necesaria desde las ciencias sociales. En efecto, en opinión de Mateo (2003) la complejidad está posibilitando metodologías no lineales aplicadas a la psicología. De esta manera la incorporación del factor tiempo es fundamental para la psicología social (McGrath \&
Tschan, 2004), lo cual ha derivado en un renovado interés por diseños longitudinales. Dada la naturaleza dinámica del objeto de estudio de la disciplina, se incide en la conveniencia de la aplicación del PC a la psicología social de la mano de autores como Munné (1993, 1994, 1995, 2004, 2005).

Según Jackson (2005), gracias a su perspectiva holística el PC constituye una descripción más completa de los procesos psicosociales ya que el comportamiento humano se definiría por las complejas relaciones entre componentes (Wagensberg, 1985). La complejidad está alumbrando la investigación de fenómenos psicosociales de constructos como el self (Codina, 2005) y está siendo aplicada al estudio de las organizaciones laborales concebidas como sistemas adaptativos complejos (Guastello, 1995, 1998; Navarro \& Quijano, 2003). Asimismo, también es asumible la psicología construccional desde la complejidad, tal y como evidencia Gergen (1996). Basta citar como ejemplos de interés los trabajos en psicología social crítica (Ibáñez, 1997; Iñiguez, 2003; Ovejero \& Ramos, 2011; Wexler, 1983), así como destacan aplicaciones a cuestiones propias de humanismo científico en cuestiones de salud (Toro \& Bareño, 2009) o propias del campo de estudio e intervención de la psicología comunitaria (Andrade, 2012).

El reconocimiento de la complejidad de la mano de Luhmann (1990) se vincula a la idea de sobreabundancia de relaciones, de posibilidades y de conexiones, de ahí la necesidad de superar la correspondencia biunívoca y lineal de elemento con elemento. De este modo, se tiende a la elaboración de modelos de fluidez social (García Selgas \& García, 2014), atendiendo a los cambios operados en los procesos sociales, productivos, técnicos, etc., en virtud de los cuales se tiende a determinar la conveniencia de describir los elementos fundamentales de las sociedades contemporáneas como fluidificados, compuestos de redes y ensamblajes (Baumann, 2000; Millar \& Page, 2007) priorizando las relaciones y trayectorias que trazan los flujos ensamblados, por encima del estudio parcializado de sus elementos básicos constitutivos (individuos, clases, estructuras, Estados, etc.).

En suma, las descripciones en ciencias sociales no son descripciones simples ni neutras, sino que resultan de nuestro diálogo con la realidad, de nuestra construcción social de la misma y de nuestros modos de acción e investigación. De acuerdo con Prigogine (1997), desde el comienzo de la revolución científica se ha comprendido que en la experiencia no sólo se observa, sino que también se interroga. Según el dilema de Epicuro el problema de la ciencia, de la inteligibilidad de la naturaleza, es inseparable del destino de los hombres.

\section{PC aplicado a las cuestiones del método en psicología social}

Existen muchos modos de analizar la realidad social, todos ellos son perfectamente válidos, lo cual debe 
tomarse como un indicador más de la complejidad de la(s) realidad (es) objeto de análisis. En función del tipo de dominio de lo humano que se investigue $y$ de las interrelaciones entre ellos, deben seleccionarse metodologías que se adecuen óptimamente al mismo. Una metodología, compleja, dinámica y no lineal ha de ocuparse de la variabilidad del objeto de estudio de la psicología social.

Baste recordar que el calificado como "viejo paradigma" en investigación experimental por McGuire (1973), ha sido puesto en entredicho, principalmente, por las sospechas sobre la capacidad de generalización de sus resultados. De este modo, en referencia a la polémica de los dos métodos naturalismo o anti-naturalismo, Ibáñez (1994) incide en el hecho de que, frente a la concepción positivista de las ciencias sociales, se desarrolló una concepción hermenéutica, culturalista e historicista. Se estima que, dadas las características diferenciadoras del objeto social, se requiere de un método distinto del que utilizaban las ciencias naturales incidiendo en el carácter idiográfico de las sociedades y la naturaleza de los significados compartidos e históricamente construidos. Frente al empiricismo maximalista, calificado en esos términos por Íñiguez (2003), fiel a los postulados del positivismo, caben otras prácticas metodológicas posibles desde las que se privilegia una investigación psicosociológica fruto de una práctica reflexiva y con relevancia social. Lo que fue interpretado como una grieta fragmentaria en lo que ha sido una metodología dominante de corte experimentalista e individualista ha supuesto, sin embargo, una ampliación de métodos que ha resultado sumamente fructífera y revitalizante, así como más adaptada a la propia complejidad del objeto de estudio.

La experimentación es, de alguna manera, el representante legítimo de los planteamientos positivistas más radicales, el análisis de su idoneidad por parte de los filósofos de la ciencia y de los científicos sociales ha centrado uno de los debates más animados en el desarrollo del conocimiento científico durante las últimas décadas (lbáñez \& Íñiguez, 1997a, 1997b; Îñiguez, 1995; Kvale, 1992). La experimentación supone una (hiper) simplificación de la realidad que viene justificada por un desarrollo teórico que la precede y avala. Desde una perspectiva conservadora de la ciencia, Bunge (1973) estableció que el conocimiento científico se caracteriza por ser racional y objetivo, fáctico y verificable. Esta caracterización sintoniza, en buena medida, con los atributos del método cuantitativo.

Más allá de estas discusiones de base epistemológica, lo cierto es que la distinción entre metodología cuantitativa y cualitativa define dos campos de investigación en la producción del conocimiento científico que profesan distintos postulados paradigmáticos, tanto en su faceta de racionalidad, cuantificación, objetividad y verificabilidad, más propio del primero, como mediante aproximaciones comprehensivas a las discursividades e interpretaciones simbólicas en escenarios de la vida real, dirigidas por supuestos hermenéuticos, característico de la metodología cualitativa (Calero, 2000). En todo caso, coincidimos con lbáñez (1992) en que cualquier método adquiere relevancia en función de la metateoría que le sirve de base. Básicamente, la distintividad de las ciencias no se cifra tanto en los temas de que se ocupa, sino, más bien atendiendo a la perspectiva y el punto de vista desde el que los abordan.

Mientras la metodología cuantitativa persigue la predicción de los fenómenos a través del análisis de las causas y los efectos, la investigación cualitativa se apoya sobre la idea de la complejidad de la realidad y en la fidelidad de la perspectiva de los actores involucrados en esa realidad (Hernández, Fernández \& Baptista, 2004), incidiéndose, pues, en las labores de interpretación y descubrimiento de significados, enfatizándose la característica única, irrepetible y subjetiva del fenómeno explorado. Ahora bien, el auge de las metodologías cualitativas está en parte determinado, no sólo por su interés en sí mismas, sino por el propio desencanto surgido a partir del uso del experimentalismo y de metodologías cuantitativas en la investigación psicosocial (Sabucedo, D'Adamo y García, 1997), y por la consiguiente adopción de un posicionamiento crítico de un "empirismo abstracto y ramplón" (Jiménez-Burillo, 2005).

Desde una perspectiva cualitativa en investigación psicosocial se pretende comprender la realidad social, concebida como resultado de un proceso constitutivo histórico y valorándose la realidad en su cualidad de epistémica, construida por un sujeto contextualizado en interacción dialéctica con una realidad dinámica. Algunos de los principios inspiradores de los métodos cualitativos (Anguera, 1995), tales como la concepción múltiple de la realidad, la función de compresión de los fenómenos investigados, la constatación de la interrelación entre investigador y objeto de investigación, la simultaneidad de los fenómenos e interacciones mutuas o el hecho de que los valores están implícitos en la investigación, entre otros, responden a los presupuestos de la concepción de la realidad desde aproximaciones nodulares del PC.

Sigue estando vigente la tendencia a conflictuar el uso -excluyente o complementario- de las metodologías cuantitativas y cualitativas en el análisis de la realidad psicosocial objeto de estudio. últimamente, la tendencia más extendida en psicología social reconocer que, a pesar de los objetivos y procedimientos distintos en ambas metodologías, se defiende la complementariedad de los métodos (Álvaro, 1995; Anguera, 1995). De este modo, la combinación del uso de metodologías cualitativas y cuantitativas, calificada como investigación multimétodos, se apoya en su complementariedad, según Polit y Hungler (2000). Es más, en investigación social si valoramos el objeto de estudio como un sistema abierto, procesual, en permanente construcción y por necesidad contextualizado, lo convierten, según 
Castoriadis (1978), en un objeto radicalmente no formalizable (Andrea de, 2010).

En suma, atendiendo la concepción de una psicología social de base historicista, antiexperimentalista y comprometida con la acción social, como defiende Gergen (1996), los conocimientos de nuestra disciplina deberían ser aportados mediante una metodología de corte cualitativo, propia de disciplinas idiográficas, siendo la psicología social una ciencia histórica y comprensiva. Los desarrollos de una psicología social de base antipositivista e histórica, transdisciplinaria, auténticamente plural, crítica e integradora (Ibáñez, 1994; Iñiguez, 2003) han supuesto savia nueva, abriendo así el espectro de acción a métodos de evaluación integral acorde a la propia naturaleza de los problemas sociales y comunitarios. En esta tesitura, el enfoque de la complejidad constituye el marco conceptual y formal desde el que se aborda, en la ciencia contemporánea, el tratamiento de la autoorganización y la complejidad, máxime teniendo en cuenta la multiestabilidad como característica del comportamiento humano.

\section{Enfoque epistémico integrado en psicología social: Fundamentos transdisciplinares, apariencias y resistencias}

La psicología social ha sido conflictiva ya desde sus inicios. Muchos han sido los enfrentamientos (psicologistas y sociologistas, instintivistas y conductistas, positivistas y antipositivistas) a los que se añaden otros más recientes y sumamente fructíferos, a nuestro parecer, como por ejemplo, modernistas frente a postmodernistas 0 reduccionistas frente a transdisciplinares. A pesar de las buenas intenciones, si bien como ideal se aboga por la transdisciplinariedad a nivel epistémico y por una praxis desde la que se promueva la transformación y cambio social, entre los profesionales de la psicología social se impone una práctica fragmentadora. En cambio, es necesario un enfoque integrador de saberes con capacidad para funcionar de un modo sistémico con intención de una praxis social realmente emancipadora.

Indiscutiblemente, a lo largo del pasado siglo XX se ha producido una transformación radical del concepto de conocimiento y del concepto de ciencia, surgiendo un nuevo concepto de la racionalidad científica, a un nuevo paradigma epistemológico. Tal y como se defiende desde orientaciones postpositivistas (postestructuralistas, deconstruccionistas, postmodernistas, la teoría crítica, etc.) de carácter más holístico, ecológico y sistémico, se aboga por una interrelación de conceptualizaciones, un pluralismo metodológico y una superación de la territorialidad disciplinar de la explicación psicosocial.

Según Nicolescu (1996), entre los objetos de estudio hay ciertas dimensiones, "niveles de realidad" a partir de una "lógica arborescente", en los términos de Morin $(1983,1988)$ para quien la transdisciplinariedad es un esquema cognitivo que permite "atravesar" las disciplinas. La ciencia está condicionada social e históricamente, al igual que cualquier actividad que implique lo humano. Durante dos siglos se ha venido sosteniendo la neutralidad y apoliticidad de la ciencia (Arias \& Abarca, 2010), naturalizándose la forma dominante de aprender "la realidad". De este modo, se valora que la fragmentación del saber en múltiples disciplinas no es algo natural, máxime cuando se asume que en campos de conocimiento como la psicología social están gestálticamente relacionadas unas con otras. En el caso de la psicología social, han de implicarse en ese diversificado campo de estudio las prácticas sociales, la intersubjetividad, la construcción de los significados sociales, y la transformación de las estructuras sociales (lbáñez, 1998).

En un intento de superación del marco epistemológico decimonónico y desde la asunción de la complejidad, el ejercicio de la psicología social en el área problematizada de las relaciones humanas ha de servirnos para profundizar dialécticamente en el proceso de construcción de significados integrados -que sean más que un mero cúmulo de intereses adosados (Jiménez Burillo, 2005)- y con potencialidad de cambio social. La supuesta trasgresión disciplinar, con la incursión en temas transversales, forma parte de ese proceso gestante, como en el caso de las corrientes humanistas, freudomarxistas, histórico-culturales o críticas, por citar algunos ejemplos, así como las propias articulaciones entre las distintas aplicaciones de la psicología social, como fase de adaptación de los intentos de comprensión transdisciplinar.

A tenor de los cambios experimentados a lo largo del pasado siglo $X X$ en las ciencias se evidencia la conveniencia de articular nuevas propuestas epistemológicas y metodológicas de análisis en la construcción del conocimiento para tratar de responder a tales cambios profundos desde posturas no reduccionistas en el proceso de aprehensión del saber (Delgado, 2009; Follari, 2001a, 2001b; Forero, 2008; Márquez, 2007; Peñuela, 2005) en la calificada por Morin, Ciurana y Motta (2003) como "era planetaria". La investigación transdisciplinar propone una completa integración teorética y práctica, de acuerdo a la asunción de un marco epistémico amplio y una cierta metametodología que sirve para integrar conceptualmente las diferentes orientaciones de sus análisis.

De acuerdo con Aronson (2003), bajo el paradigma de la transdisciplinariedad, la producción de conocimiento no opera según la oposición "conocimiento básico/conocimiento aplicado", sino en el marco de estructuraciones dinámicas y agrupamientos heterogéneos y transitorios. Efectivamente, como se ha explicitado, según Morin (1983, 1994, 2000), desde esta lógica, se estima que los factores psicológicos, sociales, culturales, biológicos y físicos son recíprocamente interdependientes, si bien la interdisciplinariedad no conjuga de forma adecuada semejante integración. Así, el intento de teorización acerca de la noción de 
contexto complejo es fundamental en el proceso de construcción epistemológica reflexiva. La perspectiva transdisciplinar en psicología social estaría asociada a la transferencia de conocimiento y a la promoción de un diálogo constructivo crítico y permanente con el desarrollo de procesos transformadores. De este modo, abundan los estudios de la interdisciplinariedad y la transdisciplinariedad en las ciencias sociales (Follari, 2001a, 2001b; García de Berrios, 2001), específicamente en la psicología social (Estrada, 2004, 2010; Munnè, 1999).

En definitiva, nuestro acervo conceptual clásico resulta insuficiente e inadecuado para simbolizar la realidad compleja. Ha de advertirse que puede que prefijos como trans (disciplinar), meta (disciplinar), post (modernidad) u otros, frecuentemente, no son más que comodines intelectuales (Martínez, 2007). Existe el riesgo que los intentos transdisciplinares, preferentemente, se conviertan en una cuestión propia de pura disquisición terminológica cuando, en realidad, la investigación transdisciplinar debería representar una completa integración teorética y práctica.

Inteligimos la realidad traduciendo ciertas claves interpretativas y configurando nuevos signos del sistema de referencia asociados a significados que son transmitidos y reasignados mediante prácticas a través de las que se procede a la salvaguardia de las órdenes dominantes. En ese intento nos valemos de palabras, propiamente, que no sólo obedecen a ciertas concepciones, sino que mediante ellas se construyen, velan, designan e imponen realidades, pero que también son instrumentos de poder-saber con criterios de verdad, son, en suma, parafraseando a Foucault (1966). "palabras y cosas".

\section{Discusión}

Las aportaciones del PC a las ciencias sociales, y en especial a la psicología social, son múltiples y diversificadas. Como paradigma emergente desde el que se contribuya a la (re) definición de los objetos de estudio de la psicología social, es deseable la superación del realismo ingenuo y la tendencia reduccionista - sea psicologista o sociologista- y entrar en la lógica de una coherencia integral, hermenéutica y sistémica, que defina nuestro campo de conocimiento desde una postura transdisciplinar integradora. En cualquier intento comprensivo de dilucidación del concepto de la psicología social es inexcusable la vinculación a ambas disciplinas, así como defendible la pertinencia de afianzar una aproximación histórica y dialéctica.

La polarización reduccionista en el análisis del comportamiento humano no es, ni siquiera, una herencia decimonónica, sino un producto de la fragmentación del saber y la (im) posición de perspectivas dominantes como constitución sui generis del pasado siglo $\mathrm{XX}$ desde la que se concede verosimilitud a una construcción fragmentaria y reificada, como aduce Morin (1994), "obras clausuradas, pero con apariencia de verdad".
Asimismo, en el caso de disciplinas como la psicología social, se aboga por un proceso prospectivo de diseño participante de unas epistemes y unas aplicaciones con relevancia social y comunitaria, pertinentes a las demandas $y$, comprometidas con la acción social, no como algo ya dado ni proyectado de modo intransferible ni reduccionista.

Los nuevos frentes de discusión no han posibilitado el tránsito como tal del paradigma mecanicista al PC pero sí la toma de conciencia progresiva de la necesidad de reformar el pensamiento y el conocimiento tanto en ciencias empírico-naturales como en las ciencias sociales. Se abren nuevas formas de pensar la realidad, reconociendo las potencialidades transformadoras de los sistemas, así como la naturaleza sinérgica de la realidad e, idealmente, se abre camino a un pluralismo metodológico. Se aboga por un intento de huir de la "tecnologización" de la psicología social y una superación de una producción académica estándar, asumiéndose que el conocimiento está incorporado a las prácticas y a los discursos (Iñiguez, 2003). Desde corrientes como la psicología social crítica o la psicología social discursiva, entre otras, se asume que el supuesto dominio de la realidad objetiva representa un intento infructuoso, difícilmente aprehensible, mientras se defiende una investigación psicosociológica con relevancia social y fruto de una práctica reflexiva e integradora.

Dadas las implicaciones éticas y sociopolíticas de las investigaciones en ciencias sociales, en concreto, en psicología social, resulta de sumo interés abogar por la responsabilidad social y comunitaria de los profesionales así como su labor crítica, de sensibilización y socioeducativa asociada a las labores de democratización de los avances en las ciencias del comportamiento y del propio progreso científico-tecnológico desde una perspectiva interdisciplinaria. Coincidimos con Núñez (1999) en que la ciencia y la tecnología son procesos sociales, condicionados cultural e históricamente y asociados a la innovación y el desarrollo social, visión contraria a la imagen propuesta desde el paradigma lógico-positivista. No se trata, por tanto, solo de contribuir a concienciar sobre los posibles daños en investigación en ciencias sociales (Santi, 2015) o de avanzar en acuerdos sobre ética profesional como autorregulación responsable (Ceballos, 2014), sino que se trataría de evitar el ocultamiento y la tergiversación de la intrínseca condición sociopolítica de disciplinas como la psicología social.

Sostenemos que lo fundamental es seleccionar los procedimientos idóneos para el problema concreto que sea objeto de investigación y contemplar las implicaciones sociales, éticas y políticas de los planteamientos a nivel epistémico y de los procedimientos metodológicos seleccionados. Si, como hace décadas manifestó Gödel (1962), no existe ningún metalenguaje universal que ponga a prueba un discurso, tampoco existe ningún instrumento $u$ operador que ponga a prueba un hecho, según la apreciación de Heisenberg (1925). 
En este sentido, el divulgador científico tiende a ocuparse de fenómenos y teorías que gozan de aceptación, la especulación con libertad a la que alude Eysenk (1977) en psicología: "hechos y palabrería", si bien debemos profundizar en las posibilidades inherentes a una psicología no especulativa ni reduccionista. La sugerente interrogación de Munnè (2007), "¿la explicación del comportamiento humano debe ser lo más simple o lo más compleja?" elicita una reflexión sobre el emergente paradigma epistemológico en las ciencias del comportamiento individual y social. En definitiva, el PC ofrece como una vía comprensiva de análisis de lo psicosocial en sus múltiples vertientes e interconexiones. Como concluye magistralmente Munnè (2007, pp. 9-10):

"A la vista de todo lo expuesto, ies arriesgado concluir que la psicología y la psicología social se están construyendo en condiciones epistemológicamente adversas? Tanto la investigación como la intervención en comportamiento humano orientadas por la simplicidad extreman (polarizan) los fenómenos y exageran (simplifican) la realidad, generando muchos más problemas que los que tratan de resolver. Sólo en la medida en que se encuentran explicaciones lo más complejas posible podrá profundizarse en el conocimiento y el tratamiento científicos del ser humano. Y para que las ciencias del comportamiento no se alejen de la realidad, asuman la incertidumbre y puedan avanzar en tales explicaciones deben reconocer paradójicamente, en un acto de humildad, que los seres humanos somos (afortunadamente) menos predecibles, controlables y regulables de lo que presupone la ciencia lineal y las explicaciones basadas en ella."

\section{Referencias}

Álvaro, J. (1995). Psicología social: perspectivas teóricas y metodológicas. Madrid: Siglo XXI.

Andrade, J. (2012). Psicología Comunitaria y clínica social. Acercamiento desde un escenario de complejidad. Revista de Psicología GEPU, 3(2), 158-175. Recuperado de https://dialnet.unirioja.es/descarga/articulo/4392 294.pdf

Andrea de, N. (2010). Perspectivas cualitativa y cuantitativa en investigación ¿inconmensurables?. Fundamentos en Humanidades, 11(1), 53-66. Recuperado http://fundamentos.unsl.edu.ar/pdf/articulo-2153.pdf

Anguera, M. (1995). Metodología cualitativa. En M. Anguera, J. Arnau, M. Ato, R. Martínez, J. Pascual \& G. Vallejo (Eds.), Métodos de investigación en psicología (pp. 513-522). Madrid: Síntesis.

Arias, L. \& Abarca, O. (2010). Algunas consideraciones en torno a la transdisciplinariedad y a la educación superior. Revista Electrónica Educare, 14(2), 7$34 . \quad$ Recuperado de https://dialnet.unirioja.es/servlet/articulo?codigo $=3641870$
Aronson, P. (2003). La emergencia de la ciencia transdisciplinar. Cinta de Moebio, 18, 2-14. Recuperado

de http://www.redalyc.org/articulo.oa?id=10101803

Bauman, Z. (2000). Liquid Modernity. Cambridge: Polity.

Bunge, M. (1973). La ciencia, su método y su filosofía. Buenos Aires: Siglo Veinte.

Byrne, D. (1998). Complexity Theory in the Social Sciences: An Introduction. New York: Routledge.

Calero, J. (2000). Investigación cualitativa y cuantitativa. Problemas no resueltos en los debates actuales. Revista Cubana de Endocrinología, 11(3), 192$198 . \quad$ Recuperado de http://www.bvs.sld.cu/revistas/end/vol11 3 00/e nd09300.htm

Carpintero, H. (2000). El hombre en el tercer milenio. En F. Ayala, H. Carpintero, P.M. Churchland, C. López, J. Maddox, J. Mira, F. Sánchez \& S. Zeki (Coords.), Ciencia y Sociedad. Nuevos enigmas científicos (pp. 141-151). Oviedo: Nobel.

Carver, C. (1997). Dynamical social psychology: Chaos and catastrophe for all. Psychological Inquiry, 8(2), 100-119. doi:10.1207/s15327965pli0802_4

Castoriadis, C. (1978). Les Carreíours du Labyrínthe. París: Seuil.

Ceballos, J. (2014). Hacia el esbozo de una ética profesional. Miscelánea Comillas: Revista de Ciencias Humanas y Sociales, 72(140-141), 159$167 . \quad$ Recuperado de http://revistas.upcomillas.es/index.php/miscelan eacomillas/article/view/5470

Codina, N. (2005). La complejidad del self y análisis empírico de su borrosidad. Encuentros en Psicología social, 3(2), 36-44. Recuperado de https://www.researchgate.net/publication/25896 5392 La complejidad del self y analisis empi rico de su borrosidad

Crespo, E. (1995). Introducción a la Psicología social. Madrid: Universitas.

Cronbach, L. (1988). Playing with chaos. Educational Researcher, 17(6), 46-49. doi:10.3102/0013189X017006046

Delgado, R. (2009). La integración de los saberes bajo el enfoque dialéctico globalizador: la interdisciplinariedad y transdisciplinariedad en educación. Investigación y postgrado, 24(3), 11$44 . \quad$ Recuperado de http://www.redalyc. org/articulo.oa?id $=65818200$ $\underline{002}$

Eldregde, N. \& Gould, S. (1972). Punctuated equilibria: An alternative to phyletic gradualism. En T. Schopf (Ed.), Models in paleobiology (pp. 82-115). San Francisco: Freeman Cooper.

Estrada, A. (2004). La psicología social en el concierto de la transdisciplinariedad. Retos latinoamericanos. Revista de Estudios Sociales, 18, 51-58. Recuperado de https://dialnet.unirioja.es/servlet/articulo?codigo $=2349203$ 
Estrada, A. (2010). Recursos crítico-interpretativos para la psicología social. Revista Colombiana de Psicología, 19(2), 261-270. Recuperado de https://dialnet.unirioja.es/servlet/articulo?codigo $=3641514$

Eysenck, H. (1977). Psicología: hechos y palabrería. Madrid: Alianza Editorial.

Fiske, S., Gilbert, D. \& Lindzey, G. (Coords.). (2010). Handbook of Social Psychology (5th ed.). New York: McGraw-Hill.

Follari, R. (2001a). Estudios culturales, transdisciplinariedad. ¿Hegemonismo en las ciencias sociales latinoamericanas?. Utopía y Praxis Latinoamericana, 6(14), 40-43. Recuperado

de

https://dialnet.unirioja.es/servlet/articulo?codigo $=2731290$

Follari, R. A. (2001b). Relevo en las ciencias sociales latinoamericanas: Estudios culturales transdisciplinariedad y multidisciplinariedad. Diálogos de la comunicación, 63, 31-36. Recuperado de http://dialogosfelafacs.net/wpcontent/uploads/2015/74/74-revista-dialogosrelevo-en-las-ciencias-sociales.pdf

Forero, Z. (2008). Atravesando disciplinas: la institucionalización de los estudios culturales en Colombia. Hispanic Research Journal: Iberian and Latin American Studies, 9(1), 65-86. Recuperado de https://dialnet.unirioja.es/servlet/articulo?codigo $=2733990$

Foucault, M. (1966). Les mots et les choses. Une archéologie des sciences humaines. Paris: Gallimard.

García de Berrios, O. (2001). Pensamiento Complejo e Interdisciplinariedad en Organizaciones Sociales: Vigencia de la Concepción Sistémica. Educere: Revista Venezolana de Educación, 12, 13-18. Recuperado de https://dialnet.unirioja.es/servlet/articulo?codigo $=3650424$

García, F. \& García, A. (2014). Hacia la elaboración de modelos de la fluidez social I: Teoría de la fluidez social y teorías de la complejidad. Atenea Digital, 14(2), 203-226. Recuperado de http://atheneadigital.net/article/view/v14-n2garcia-garcia

Gell-Mann, M. (1998). El Quark y el Jaguar. Aventuras en lo simple y lo complejo. Barcelona: Tusquets.

Gergen, K. (1996). La construcción social: emergencia y potencial. En M. Pakman (Comp.), Construcciones de la experiencia humana (pp. 139-182). Barcelona: Gedisa.

Gödel, K. (1962). On formally undecidable propositions in Principia mathematica and related systems. New York: Basic Books.

Graumann, C. (1990). Introducción a una historia de la Psicología social. En M. Hewstone, W. Strobe, J. Codol \& G. Stephenson (Eds.), Introducción a la Psicología social. Una perspectiva europea (pp. 21-35). Barcelona: Ariel.
Gribbin, J. (2006). Así de simple. El caos, la complejidad y la aparición de la vida. Barcelona: Crítica.

Guastello, S. (1995). Chaos, catastrophe and human affairs: Applications of nonlinear dynamics to work, organizations and social evolution. Hillsdale, NJ: Lawrence Erlbaum.

Guastello, S. (1998). Creative problem solving groups at the edge of chaos. Journal of Creative Behavior, $32(1), \quad 38-57 . \quad$ doi:10.1002/j.21626057.1998.tb00805.x

Heisenberg, W. (1925). Über Quantentheoretischen Umdeutung Kinematischer und Mechanischer Beziehungen. Zeitschrift für Logik, 33, 879-893.

Hernández Sampieri, R., Fernández, C. \& Baptista, P. (2004). Metodología de la investigación. Santiago, Chile: McGraw-Hill.

Holland, J. (1995). Hidden Order: How Adaptation Builds Complexity. Reading: Addison-Wesley.

Holland, J. (1998). Emergence: From Chaos to Order. Oxford: Oxford University Press.

Ibáñez, J. (1992). Más allá de la Sociología. El grupo de discusión: Técnica y crítica. Madrid: Siglo XXI.

Ibáñez, J. (1993). El papel del sujeto en la teoría (hacia una sociología reflexiva). En E. Lamo de Espinosa \& J. Rodríguez (Coords.), Problemas de teoría social contemporánea (pp. 359-386). Madrid: CIS.

Ibáñez, J. (1998). Las paradojas de la investigación social: una tarea necesaria e imposible. En J. Ibáñez (Coord.), Nuevos avances en la investigación social. La investigación social de segundo orden (Vol. 1, pp. 178-187). Barcelona: Suplementos Anthropos, 22.

Ibáñez, T. (1994). Psicología social Construccionista. México: Dirección de Publicaciones de la Universidad de Guadalajara.

Ibáñez, T. (1996). Fluctuaciones conceptuales en torno a la postmodernidad y la psicología. Caracas: Universidad Central de Venezuela.

Ibáñez, T. (1997). Why a critical social psychology? En T. Ibáñez \& L. Íñiguez (Eds.), Critical social psychology (pp. 27-41). London: Sage.

Ibáñez, T. (2004). El por qué y el cómo de la psicología social. En T. Ibáñez (Coord.), Introducción a la Psicología social (pp. 53-91). Barcelona: UOC.

Ibáñez, T. \& Íñiguez, L. (1997a). Aspectos metodológicos de la Psicología social Aplicada. En J. Álvaro, J. Torregrosa \& A. Garrido (Eds.), Psicología social Aplicada (pp. 57-82). Madrid: McGraw-Hill.

lbáñez, T. \& Íñiguez, L. (1997b). Critical Social Psychology. London: Sage.

Íñiguez, L. (2003). La psicología social como crítica: Continuismo, estabilidad y efervescencias. Tres décadas después de la "Crisis". Revista Interamericana de Psicología, 37(2), 221-238. Recuperado de http://www.psicorip.org/Resumos/PerP/RIP/RIP 036a0/RIP03717.pdf 
Jackson, P. (2005). Indigenous theorizing in a complex world. Asian Journal of Social Psychology, 8, 5164. doi:10.1111/j.1467-839X.2005.00156.X

Jiménez-Burillo, F. (2005). Contribución a la crítica de la psicosociología imperante. Encuentros en Psicología social, 3, 5-31.

Johnson, S. (2003). Sistemas emergentes. O que tienen en común hormigas, neuronas, ciudades y software. Barcelona: Fondo de Cultura Económica.

Kelly, B. (1981). Inventing Psychology's Past: E.G. Boring's Historiography in Relation to the Psychology of his time. The Journal of Mind and Behavior, 2(3), 229-241. Recuperado de https://www.jstor.org/stable/43852866?seq=1\#p age scan tab contents

Klappenbach, H. (2006). Construcción de tradiciones hstoriográficas en Psicología y Psicoanálisis. Psicologia em Estudo, Maringá, 11(1). 3-17. doi:10.1590/S1413-73722006000100002

Kvale, S. (Ed.). (1992). Psychology and postmodernism. London: Sage.

Lefebvre, G. (1974). El nacimiento de la historiografía moderna. Barcelona: Martínez-Roca.

Lewin, R. (1995). Complejidad. El caos como generador del orden. Barcelona: Tusquets Editores.

Lord, F. \& Novick, M. (1968). Statistical theories of mental test scores. Reading: Addison Wesley.

Lorenz, E. (1965/1995). La esencia del caos. Un cambio de conocimiento que se ha convertido en parte importante del mundo que nos rodea. Madrid: Debate.

Luengo-González, E. (2008). La simplicidad del método científico y la complejidad de lo real. En R. Lanz \& R. Reynoso (Coords.), Ni una sola ciencia, ni una sola técnica, debate abierto sobre misión ciencia (Tomo 3, pp. 1-33). Caracas: Ministerio de Ciencia y Tecnología de Venezuela.

Luhmann, N. (1990). Sociedad y sistema: la ambición de la teoría. Barcelona: Paidós.

Luhmann, N. (1998). Complejidad y modernidad, de la unidad a la diferencia. Madrid: Trotta.

Mandelbrot, B. (1987). Los objetos fractales. Barcelona: Tusquets.

Márquez, A. (2007). Pensar la Complejidad desde la praxis cognoscente de la racionalidad intersubjetiva. Utopía y praxis latinoamericana: Revista Internacional de Filosofía Iberoamericana $y$ Teoría Social, 38, 99-106. Recuperado de https://dialnet.unirioja.es/servlet/articulo?codigo $=2373918$

Martín-Baró, I. (1983). Acción e ideología. Psicología social desde Centroamérica I. San Salvador: UCA Editores.

Martínez, M. (2007). Conceptualización de la transdisciplinariedad. Polis, 5(16), 5-35. Recuperado de https://polis.revues.org/4623

Mateo, M. (2003). Notas sobre la complejidad en la Psicología. Anales de Psicología, 19(2), 315-
326.

Recuperado

de

http://www.redalyc. org/articulo.oa?id=16719212

Maturana, H. \& Varela, F. (1990). El árbol del conocimiento: las bases biológicas del conocimiento humano. Madrid: Debate.

Maturana, H. \& Varela, F. (1994). De máquinas y seres vivos: Autopoiesis, la organización de lo vivo. Santiago, Chile: Editorial Universitaria.

McGrath, J. \& Tschan, F. (2004). Temporal matters in social psychology: Examining the role of time in the lives of groups and individuals. Washington: American Psychological Association.

McGuire, W. (1973). The yin and yang of progress in social psychology: Seven Koan. Journal of Personality and Social Psychology, 26, 446-456. doi:10.1177/053901893032004001

Miller, J. \& Page, S. (2007). Complex adaptive systems. Princeton: Princeton University Press.

Montero, M. (2010). Crítica, autocrítica y construcción de teoría en la psicología social latinoamericana. Revista Colombiana de Psicología, 19(2), 177$191 . \quad$ Recuperado de http://www.redalyc. org/articulo.oa?id=80415435 $\underline{003}$

Moral, M. \& Ovejero, A. (2013). Del Interaccionismo Simbólico a la Etnometodología: conceptos fundacionales, diversificaciones e influencias. Revista de Psicología social y Personalidad, 29(1), 1-24. Recuperado de http://www.amepso.org/files/downs fR3Wim8W 7 uij2Vy.pdf

Morin E. (1983). Interdisciplinariedad y ciencias humana. Madrid: Tecnos/UNESCO.

Morin, E. (1988). El conocimiento del conocimiento. Madrid: Cátedra.

Morin, E. (1994). El conocimiento del conocimiento. El método. Madrid: Cátedra.

Morin, E. (1995). Introducción al pensamiento complejo. Barcelona: Gedisa.

Morin, E. (1999). La cabeza bien puesta. Repensar la Reforma. Reformar el pensamiento. Buenos Aires: Nueva Visión.

Morin, E. (2001). Los Siete saberes necesarios para la educación del futuro. Buenos Aires: Nueva Visión.

Morin, E., Ciurana, E. \& Motta, R. (2003). Educar en la era planetaria. Barcelona: Gedisa.

Munné, F. (1993). La teoría del caos y la psicología social. Un nuevo enfoque epistemológico para el comportamiento social. En I. Fernández Jiménez \& M. Martínez (Comps.), Epistemología y procesos psicosociales básicos (pp. 37-48). Sevilla: Eudema.

Munné, F. (1994). Complejidad y caos: Más allá de una ideología del orden y desorden. En M. Montero (Coord.), Conocimiento, realidad e ideología (pp. 9-18). Caracas: AVEPSO. 
Munné, F. (1995). Las teorías de la complejidad y sus implicaciones en las ciencias del comportamiento. Revista Interamericana de Psicología, 29, 1-12.

Munnè, F. (2004). El retorno de la Complejidad y la nueva imagen del ser humano: hacia una Psicología Compleja. Revista Interamericana de Psicología/Interamerican Journal of Psychology, 38(1), 23-31. Recuperado de http://www.redalyc.org/pdf/284/28438104.pdf

Munné, F. (2005). Número monográfico: ¿Qué es la complejidad? Encuentros en Psicología social, 3(2), 6-17.

Munné, F. (2007). ¿La explicación del comportamiento humano debe ser lo más simple o lo más compleja? Encuentros en Psicología social, 4, 310.

Navarro, J. \& Quijano, S. (2003). Dinámica no-lineal en la motivación en el trabajo: propuesta de un modelo y resultados preliminares. Psicothema, 15(4), 643-649. Recuperado de http://www.psicothema.com/psicothema.asp?id= 1118

Nicolescu, B. (1996). La transdisciplinarité manifeste. Mónaco: Éditiones du Rocher.

Nicolescu, B. (2002). Manifesto of Transdisciplinarity. Albany: State University of New York Press.

Nicolis, G. \& Prigogine, I. (1994). La estructura de lo complejo: en el camino hacia una nueva comprensión de las ciencias. Madrid: Debate.

Núñez, J. (1999). La ciencia y la tecnología como procesos sociales. Lo que la educación científica no debería olvidar. La Habana: Félix Varela.

Ovejero, A. \& Ramos, J. (Eds.). (2011). Psicología social Crítica. Madrid: Biblioteca Nueva.

Pastor, J. \& García-Izquierdo, A. (2007). Complejidad y Psicología social de las Organizaciones. Psicothema, 19(2), 212-217. Recuperado de http://www.redalyc.org/pdf/727/72719205.pdf

Peñuela, L. (2005). La transdiciplinariedad. Más allá de los conceptos, la dialéctica. Andamios, 1(2), 43-77. Recuperado http://www.redalyc. org/articulo.oa?id=62810203

Poincaré, H. (1908/1948). Ciencia y método. Madrid: Espasa-Calpe.

Polit, D. \& Hungler, B. (2000). Investigación científica en Ciencias de la salud. México: McGraw-Hill Interamericana.

Prigogine, I. (1983). ¿Tan sólo una ilusión? Una exploración del caos al orden. Barcelona: Tusquets.

Prigogine, I. (1997). El fin de las certidumbres. Madrid: Taurus.

Sabucedo, J., D'Adamo, O. \& García, V. (1997). Fundamentos de psicología social. Madrid: Siglo XXI.
Santi, M. (2015). El debate sobre los daños en investigación en ciencias sociales. Revista de bioética y derecho: publicaciones del Máster en Bioética y Derecho, 34, 11-25. doi:10.1344/rbd2015.34.12063

Stewart, I. (1991). ¿Juega Dios a los dados? La nueva matemática del caos. Barcelona: Crítica.

Stoking, G. (1965). On the limits of presentism and historicism in the historiography of the behavioral sciences. Journal of the history of the behavioral sciences, 1, 211-219. doi:10.1002/15206696(196507)1:3<211::AIDJHBS2300010302>3.0.CO;2-W

Thom, R. (1997). Estabilidad estructural y morfogénesis. Barcelona: Gedisa.

Toro, F. \& Bareño, J. (2009). Humanismo científico, calidad en salud y complejidad. Revista CES Medicina, 23(2), 91-98. Recuperado de http://www.redalyc. org/articulo.oa?id=26112035 5010

Wagensberg, J. (1985). Ideas sobre la complejidad del mundo. Barcelona: Tusquets.

Wexler, P. (1983). Critical Social Psychology. Boston: Routledge and Kegan Paul.

Woodward, W. (1980). Toward a Critical Historiography of Psychology. En J. Brozek \& L. Pongratz (Eds.), Historiography of Modern Psychology (pp. 2754). Toronto/Göttingen: Hogrefe.

Woolgar, S. (Ed.). (1988). Ciencia: abriendo la caja negra. Barcelona: Anthropos.

Zadeh, L. (1965). Fuzzy sets. Information and control, 8, 338-353. doi:10.1016/S0019-9958(65)90241-X

Zimmerman, B. (1993). Chaos \& Nonequilibrium: the flip side of strategic processes. Organization Development Journal, 11(1), 31-38. Recuperado de http://psycnet.apa.org/psycinfo/1993-39479001 